TI 2018-004/III

Tinbergen Institute Discussion Paper

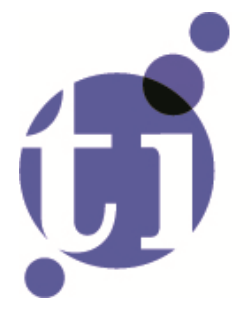

tinbergen
institute

\title{
Management Information, Decision Sciences, and Financial Economics: A Connection
}

Chia-Lin Chang ${ }^{1}$

Michael McAleer ${ }^{2}$

Wing-Keung Wong ${ }^{3}$

1: Department of Applied Economics, Department of Finance, National Chung Hsing University, Taiwan

2: Department of Finance, Asia University, Taiwan; Discipline of Business Analytics, University of Sydney Business School, Australia; Econometric Institute, Erasmus School of Economics, Erasmus University Rotterdam, The Netherlands; Department of Quantitative Analysis, Complutense University of Madrid, Spain; Institute of Advanced Sciences, Yokohama National University, Japan

3: Department of Finance and Big Data Research Center, Asia University, Taiwan; Department of Economics and Finance, Hang Seng Management College, Hong Kong, China; Department of Economics, Lingnan University, Hong Kong, China 
Tinbergen Institute is the graduate school and research institute in economics of Erasmus University Rotterdam, the University of Amsterdam and VU University Amsterdam.

Contact: discussionpapers@tinbergen.nl

More TI discussion papers can be downloaded at the Tinbergen Site

Tinbergen Institute has two locations:

Tinbergen Institute Amsterdam

Gustav Mahlerplein 117

1082 MS Amsterdam

The Netherlands

Tel.: +31(0)20 5984580

Tinbergen Institute Rotterdam

Burg. Oudlaan 50

3062 PA Rotterdam

The Netherlands

Tel.: +31(0)10408 8900 


\title{
Management Information, Decision Sciences, and Financial Economics: A Connection*
}

\author{
Chia-Lin Chang \\ Department of Applied Economics \\ Department of Finance \\ National Chung Hsing University, Taiwan \\ Michael McAleer \\ Department of Finance \\ Asia University, Taiwan \\ and \\ Discipline of Business Analytics \\ University of Sydney Business School, Australia \\ and \\ Econometric Institute, Erasmus School of Economics \\ Erasmus University Rotterdam, The Netherlands \\ and \\ Department of Quantitative Economics \\ Complutense University of Madrid, Spain \\ and \\ Institute of Advanced Sciences \\ Yokohama National University, Japan \\ Wing-Keung Wong \\ Department of Finance and Big Data Research Center \\ Asia University, Taiwan \\ and \\ Department of Economics and Finance \\ Hang Seng Management College, Hong Kong, China \\ and \\ Department of Economics \\ Lingnan University, Hong Kong, China
}

January 2018

* For financial and research support, the first author is grateful to the National Science Council, Ministry of Science and Technology (MOST), Taiwan, the second author wishes to thank the Australian Research Council and the National Science Council, Ministry of Science and Technology (MOST), Taiwan, and the third author acknowledges the Research Grants Council of Hong Kong, Asia University, Hang Seng Management College, Lingnan University, and the National Science Council, Ministry of Science and Technology (MOST), Taiwan. The third author would also like to thank Robert B. Miller and Howard E. Thompson for their continuous guidance and encouragement. 


\begin{abstract}
The paper provides a brief review of the connecting literature in management information, decision sciences, and financial economics, and discusses some research that is related to the three cognate disciplines. Academics could develop theoretical models and subsequent econometric models to estimate the parameters in the associated models, and analyze some interesting issues in the three related disciplines.
\end{abstract}

Keywords: Management information, decision sciences, financial economics, theoretical models, econometric models.

JEL: A10, G00, G31, O32, 


\section{Introduction}

There are many studies that link management information, decision sciences, and financial economics. In this paper, we discuss different types of utility functions, stochastic dominance (SD), mean-risk (MR) models, portfolio optimization (PO), and other behavioral financial economics as these topics are popular in management information, decision sciences, and financial economics. Academics could develop theoretical models and thereafter develop econometric models to estimate the associated parameters to analyze some interesting issues in management information, decision sciences, and financial economics.

The plan of the remainder of the paper is as follows. In Section 2, some theoretical models are discussed. Alternative statistical and econometrics models are analyzed in Section 3. A brief discussion of empirical models is presented in Section 4. Some concluding remarks are given in Section 5 .

\section{Theoretical Models}

It is important to commence any rigorous research in management information, decision sciences, and financial economics by developing appropriate theoretical models. We have been developing some theories to extend those that have been discussed in a number of existing literature reviews. We discuss some of our research in the following subsections.

\subsection{Cost of capital}

Gordon and Shapiro (1956) first equate the price of a share with the present value of future dividends and derived the well-known relationship. Subsequently, there have been many improvements on the theory. For example, Thompson (1985) combines the 'dividend yield plus growth' method with Box-Jenkins time series analysis of past dividend experience to estimate the cost of capital and its 'reliability' for individual firms. Thompson and Wong (1991) estimate 
the cost of capital using discounted cash flow (DCF) methods requires forecasting dividends. They show that past dividend behavior may support more than one reasonable forecasting model and that reasonable forecasting models can lead to divergent estimates of the cost of capital. They also find no way of selecting the best cost of capital estimate. Any choice must ultimately be based on the 'judgment' of the analyst.

Thompson and Wong (1996) extend Thompson's work using time series models within the discounted cash flow framework to estimate the cost of equity capital for a firm. In particular, they prove the existence and uniqueness of a solution for the cost of equity capital. They also show that the cost of equity function is continuously differentiable and derive the formula for its reliability.

However, their approaches cannot be used if 'reliability' does not exist, or if there are multiple solutions for the 'reliability'. Wong and Chan (2004) extend their theory by proving the existence and uniqueness of this reliability. In addition, they propose estimators for the reliability and prove that the estimators converge to a true parameter. The estimation approach is further simplified, hence rendering computation easier. In addition, the properties of the cost of capital and its reliability will be analysed with illustrations of several commonly used Box-Jenkins models.

We have been applying the theory of cost of capital to develop investors' behavioral models that can be used to explain market volatility, under- and overreaction and their magnitude effects as discussed in next subsection.

\subsection{Investors' behavioral models by using cost of capital and conservative and representative heuristics}

We have been developing some behavioral models by using the theory of cost of capital and conservative and representative heuristics, and develop a new Bayesian approach that can be used to explain some financial anomalies including market volatility, the short-run 
underreaction, long-run overreaction, and their magnitude effects. We then develop some statistics to test the existence of the financial anomalies.

There has been considerable interest in the Bayesian approach for explaining investors' behavioral biases by incorporating conservative and representative heuristics when making financial decisions. For example, Barberis, Shleifer, and Vishny (1998) and others have developed Bayesian models to explain investors' behavioral biases by using conservatism heuristics and the representativeness heuristics in making decisions. To extend their work and to establish a quantitative link between some important market anomalies and investors' behavioral biases, Lam,

Liu, and Wong (2010) develop a model of weight assignments using a pseudo-Bayesian approach that reflects investors' behavioral biases. In this parsimonious model of investor sentiment, weights induced by investors' conservative and representative heuristics are assigned to observations of the earning shocks of stock prices. Such weight assignments enable us to provide a quantitative link between some market anomalies and investors' behavioral biases. The seriousness of an anomaly can be quantitatively assessed by investigating into its dependency on weights. New results other than the short-run underreaction and long-run overreaction can be derived, and new hypotheses can be formed.

Lam, Liu, and Wong (2012) further extend their work by developing properties to explain some market anomalies, including short-term underreaction, long-term overreaction, and excess volatility. We also explain in details the linkage between these market anomalies and investors' behavioral biases.

In response to the recent global financial crisis, Fung, Lam, Siu, and Wong (2011) introduce a new pseudo-Bayesian model to incorporate the impact of a financial crisis. Properties of stock returns during the financial crisis and recovery from the crisis are established. The proposed model can be applied to investigate some important market anomalies, including short-term underreaction, long-term overreaction, and excess volatility during financial crisis. We also explain in some detail the linkage between these market anomalies and investors' behavioral biases during a financial crisis. 
Guo, McAleer, Wong, and Zhu (2017) introduce a new Bayesian approach to explain some market anomalies during financial crises and subsequent recovery. We assume that the earnings shock of an asset follows a random walk model with and without drift to incorporate the impact of financial crises. We further assume the earning shock follows an exponential family distribution to take care of symmetric as well as asymmetric information. By using this model setting, they develop some properties on the expected earnings shock and its volatility, and establish properties of investor behavior on the stock price and its volatility during financial crises and subsequent recovery. Thereafter, we develop properties to explain excess volatility, short-term underreaction, long-term overreaction, and their magnitude effects during financial crises and subsequent recovery.

\subsection{Modeling different types of investors}

We have been developing some theories, estimation, and testing to study different utility functions and the preferences of different types of investors. We summarize some of the results here. Readers may refer to Sriboonchitta, Wong, Dhompongsa, and Nguyen (2009) and Bai, Guo, Li, and Wong, (2017) for further information.

\subsubsection{Different Types of Utility Functions}

Within the optimal production and hedging decision framework, Lien (2008) compares the exponential utility function with its second-order approximation under the normality distributional assumption. Guo, Lien, and Wong (2016) extend the result by comparing the exponential utility function with a 2 n-order approximation for any integer $n$. We then propose an approach with an illustration to find the smallest $\mathrm{n}$ that provides a suitable approximation.

\subsubsection{Stochastic Dominance}

We have been developing several theories in stochastic dominance, and discuss some here. 


\subsubsection{Behavior of risk averters and risk seekers}

Wong and Li (1999) develop some theoretical results to compare the preferences of different combinations of several assets for risk averters and risk seekers. We also compare the preferences between a convex combination of several continuous distributions and a single continuous distribution. Wong (2007) extends the stochastic dominance (SD) theory by introducing the first three orders of both ascending SD (ASD) and descending SD (DSD) to decisions in business planning and investment to risk-averse and risk-loving decision makers so that they can compare both returns and loss.

We provide investors with more tools for empirical analysis, with which they can identify the first order ASD and DSD prospects and discern arbitrage opportunities that could increase their utility as well as wealth, and create a zero-dollar portfolio to make huge profits. Our tools also enable investors and business planners to identify the third-order ASD and DSD prospects and make better choices. To complement the stochastic dominance approach, we also introduce an improved mean-variance criterion to decisions in business planning or investment on both return and loss for risk-averse and risk-loving investors. We then illustrate the superiority of the present approaches with well-known examples in the literature, and discuss the relationship between the improved stochastic dominance and mean-variance criteria.

Chan, Clark, and Wong (2012) analyze properties of stochastic dominance (SD) for risk-averse investors and risk-seeking SD (RSD) for risk-seeking investors which, in turn, enables us to study their behavior. We first discuss the basic property of SD and RSD linking the SD and RSD to expected-utility maximization. Thereafter, we prove that a hierarchy exists in both SD and RSD relationships and that the higher orders of SD and RSD can be inferred by the lower orders of SD and RSD, but not vice-versa. Furthermore, we study the conditions in which third-order SD preferences are 'the opposite of' or 'the same as' their counterpart third-order RSD preferences.

In addition, we establish the relationship between the order of the variances and that of the integrals for two assets, which enables us to establish certain relationships between the 
dominance of the variances and the second- and third-order SD and RSD for two assets under the condition of equal means. The theory developed in this paper provides a set of tools that enables investors to identify prospects for first-, second-, and third-order SD and RSD, and thereby enables investors to improve their investment decisions. Another contribution in this paper is that we recommend checking the dominance of the means of the distributions to draw inference of the preference for two different assets for third-order risk averters and risk seekers. We illustrate this idea by comparing the investment behaviors of both third-order risk averters and risk seekers in bonds and stocks.

Guo and Wong (2016) extend some well-known univariate stochastic dominance results to multivariate stochastic dominance (MSD) for both risk averters and risk seekers, respectively, to $\mathrm{n}$ order for any $\mathrm{n}>0$, when the attributes are assumed to be independent and the utility is assumed to be additively and separable. Under these assumptions, we develop some properties for MSD for both risk averters and risk seekers. For example, we prove that MSD are equivalent to the expected-utility maximization for both risk averters and risk seekers, respectively.

We show that the hierarchical relationship exists for MSD, establish some dual relationships between the MSD for risk averters and risk seekers, develop some properties for non-negative combinations and convex combinations random variables of MSD, and develop the theory of MSD for the preferences of both risk averters and risk seekers on diversification. At last, we discuss some MSD relationships when attributes are dependent, and discuss the importance and use of the results developed in the paper.

\subsubsection{Behavior of investors with S-shaped and reversed S-shaped utility functions}

Wong and Chan (2008) extend the work on Prospect Stochastic Dominance (PSD) theory and Markowitz Stochastic Dominance theory (MSD) to the first three orders, and link the corresponding S-shaped and reverse S-shaped utility functions to the first three orders. We also provide experiments to illustrate each case of the MSD and PSD to the first three orders, and demonstrate that the higher order MSD and PSD cannot be replaced by the lower order MSD and PSD. Furthermore, we formulate the following PSD and MSD properties: hierarchy exists in 
both PSD and MSD relationships; arbitrage opportunities exist in the first orders of both PSD and MSD; and for any two prospects under certain conditions, their third order MSD preference will be "the opposite of" or "the same as" their counterpart third-order PSD preference.

\subsubsection{Behavior of investors with both risk averse and risk seeking components}

Guo, Qiao, and Wong (2017) introduce a new stochastic dominance (SD) theory for investors with $\mathrm{AD}$ utility that consists of both risk-averse and risk-seeking components. Based on the SD theory developed in the paper, we find a new solution to answer the observation posed by Friedman and Savage (1948) that people could buy insurance and try their luck with lotteries as well. We find that investors with $\mathrm{AD}$ utility will buy both insurance and try their luck with lotteries to obtain higher expected utility. We find that investors with AD utility will buy both bonds and stocks and both bonds and futures to obtain higher expected utility. In addition, we develop some properties, including expected-utility maximization, hierarchy, transitivity, and diversification, for the new SD theory for investors with AD utility.

\subsubsection{Almost Stochastic Dominance}

Guo, Zhu, Wong, and Zhu (2013) provide more information on both the expected-utility maximization and the hierarchy property. For almost stochastic dominance, Leshno and Levy (2002) propose a definition and Tzeng, Huang, and Shih (2013) modify it to provide another definition. We show that the former has the hierarchy property but not expected-utility maximization, whereas the latter has expected-utility maximization but not the hierarchy property.

Guo, Post, Wong, and Zhu (2014) establish necessary conditions for Almost Stochastic Dominance criteria of various orders. These conditions take the form of restrictions on algebraic combinations of moments of the probability distributions in question. The relevant set of conditions depends on the relevant order of ASD, but not on the critical value for the admissible violation area. These conditions can help to reduce the information requirement and computational burden in practical applications. A numerical example and an empirical 
application to historical stock market data illustrate the moment conditions. The first four moment conditions, in particular, seem appealing for many applications.

Guo, Wong, and Zhu (2016) first extend the theory of almost stochastic dominance (ASD) (for risk averters) to include the ASD for risk-seeking investors. We then study the relationship between ASD for risk seekers and ASD for risk averters. Recently, Tsetlin, Winkler, Huang, and Tzeng (2015) develop the theory of generalized ASD (GASD). We then briefly discuss the advantages and disadvantages of ASD and GASD.

\section{$2.4 \quad$ Indifference Curves}

Wong and Ma (2008) extend the work on location-scale (LS) family with general $\mathrm{n}$ random seed sources. First, we clarify and generalize existing results in this multivariate setting. Some useful geometrical and topological properties of the location-scale expected utility functions are obtained. Second, we introduce and study some general non-expected utility functions defined over the LS family. Special care is taken in characterizing the shapes of the indifference curves induced by the location-scale expected utility functions and non-expected utility functions. Finally, efforts are made to study several well-defined partial orders and dominance relations defined over the LS family. These include the first- and second-order stochastic dominance, the mean-variance rule, and a newly defined location-scale dominance.

Broll, Egozcue, Wong, and Zitikis (2010) discuss prospect theory and establish general results concerning certain covariances from which we can in turn infer properties of indifference curves and hedging decisions within the prospect theory.

\subsection{Diversification}

Wong and Li (1999) extend Fishburn (1974)'s convex stochastic dominance theorem by including any distribution function, developing the results for both risk seekers as well as risk averters, and including third-order stochastic dominance. Our results are used to extend a 
theorem of Bawa, Bodurtha Jr, Rao, and Suri (1985) on comparison between a convex combination of several continuous distributions and a single continuous distribution.

Li and Wong (1999) develop some results for the diversification preference for risk averters and risk seekers. Egozcue and Wong (2010) incorporate both majorization theory and stochastic dominance theory to develop a general theory and a unifying framework for determining the diversification preferences of risk-averse investors and conditions under which they would unanimously judge a particular asset to be superior. In particular, we develop a theory for comparing the preferences of different convex combinations of assets that characterize a portfolio to give higher expected utility by second-order stochastic dominance. Our findings also provide an additional methodology for determining the second-order stochastic dominance efficient set.

Egozcue, Fuentes García, Wong, and Zitikis (2011) study rankings of completely and partially diversified portfolios and also of specialized assets when investors follow so-called Markowitz preferences. It turns out that diversification strategies for Markowitz investors are more complex than in the case of risk-averse and risk-inclined investors, whose investment strategies have been extensively investigated in the literature.

In particular, we observe that for Markowitz investors, preferences toward risk vary depending on their sensitivities toward gains and losses. For example, it turns out that, unlike in the case of risk-averse and risk-inclined investors, Markowitz investors might prefer investing their entire wealth in just one asset. This finding helps us to better understand some financial anomalies and puzzles, such as the well-known diversification puzzle, which notes that some investors tend to concentrate on investing in only a few assets instead of choosing the seemingly more attractive complete diversification.

Lozza, Wong, Fabozzi, and Egozcue (2017) provide a general valuation of the diversification attitude of investors. First, we empirically examine the diversification behavior of mean-variance investors in the US stock market during the 11-year period 2003-2013. We then analyze the diversification problem from the perspective of risk-averse investors and risk-seeking investors. 
Second, we prove that investors' optimal choices will be similar if their utility functions are not too distant, independent of their tolerance (or aversion) to risk. Finally, we discuss investors' attitude towards diversification when the choices available to investors depend on several parameters.

\subsection{Risk Measures}

We have been developing properties for several risk measures to be used in finance, economics, and other areas. We briefly discuss the properties for some risk measures developed in this section.

\subsubsection{VaR and CvaR}

Ma and Wong (2010) establish some behavioral foundations for various types of VaR models, including $\mathrm{VaR}$ and conditional- $\mathrm{VaR}$, as measures of downside risk. We establish some logical connections among VaRs, conditional-VaR, stochastic dominance, and utility maximization. Though supported to some extents with unanimous choices by some specific groups of expected or non-expected-utility investors, VaRs as profiles of risk measures at various levels of risk tolerance are not quantifiable - they can only provide partial and incomplete risk assessments for risky prospects.

We also include in our discussion the relevant VaRs and several alternative risk measures for investors: these alternatives use somewhat weaker assumptions about risk-averse behavior by incorporating a mean-preserving-spread. For this latter group of investors, we provide arguments

for and against the standard deviation versus $\mathrm{VaR}$ and conditional-VaRs as objective and quantifiable measures of risk in the context of portfolio choice.

\subsubsection{Omega ratio}

Both stochastic dominance and Omega ratio can be used to examine whether market is efficient, whether there is any arbitrage opportunity in the market, and whether there is any anomaly in the 
market. Guo, Jiang, and Wong (2017) study the relationship between stochastic dominance and Omega ratio. They find that second-order stochastic dominance (SD) and/or second-order riskseeking SD (RSD) alone for any two prospects is not sufficient to imply Omega-ratio dominance insofar that the Omega ratio of one asset is always greater than that of the other one. We extend the theory of risk measures by proving that the preference of second-order SD implies the preference of the corresponding Omega ratios only when the return threshold is less than the mean of the higher-return asset.

On the other hand, the preference of the second-order RSD implies the preference of the corresponding Omega ratios only when the return threshold is larger than the mean of the smaller-return asset. Nonetheless, first-order SD does imply Omega-ratio dominance. Thereafter, we apply the theory developed in this paper to examine the relationship between property size and property investment in the Hong Kong real estate market. We conclude that the Hong Kong real estate market is not efficient and there are expected arbitrage opportunity and anomaly in the Hong Kong real estate market. Our findings are useful for investors and policy makers in real estate.

\subsubsection{High-order risk measures}

$\mathrm{Niu}$, Wong, and $\mathrm{Xu}$ (2017) extend the theory between the Kappa ratio and stochastic dominance (SD) and risk-seeking SD (RSD) by establishing several relationships between first- and higher-

order risk measures and (higher-order) SD and RSD. We first show the sufficient relationship between the $(n+1)$-order SD and the n-order Kappa ratio. We then discover that, in general, the necessary relationship between SD/RSD and the Kappa ratio cannot be established.

Thereafter, we find that when the variables being compared belong to the same location-scale family or the same linear combination of location-scale families, we can obtain the necessary relationships between the $(\mathrm{n}+1)$-order SD with the n-order Kappa ratio when we impose some conditions on the means. Our findings enable academics and practitioners to reach better decisions in their analysis. 


\subsubsection{Two-Moment Decision Model}

Alghalith, Guo, Niu, and Wong (2017) analyze the impacts of joint energy and output prices uncertainties on the inputs demands in a mean-variance framework. We find that an increase in expected output price will cause the risk-averse firm to increase the input demand, while an increase in expected energy price will cause the risk-averse firm to decrease the demand for energy and increase the demand for non-risky inputs. Furthermore, increasing the variance of the energy price will necessarily cause the risk-averse firm to decrease the demands for the non-risky inputs. Moreover, we investigate the two cases with only uncertain energy price and only uncertain output price. In the case with only uncertain energy price, we find that the uncertain energy price has no impact on the demands for the non-risky inputs.

Alghalith, Niu, and Wong (2017) analyze the impacts of joint energy and output prices uncertainties on the inputs demands in a mean-variance framework. We find that the concepts of elasticities and variance vulnerability play important roles in the comparative statics analysis. If the firms' preferences exhibit variance vulnerability, increasing the variance of energy price will necessarily cause the risk-averse firm to decrease the demands for the non-risky inputs.

Furthermore, we investigate two special cases with only uncertain energy price and only uncertain output price. In the case with only uncertain energy price, we find that the uncertain energy price has no impact on the demands for the non-risky inputs. Besides, if the firms' preferences exhibit variance vulnerability, increasing the variance of energy price will cause the risk-averse firm to decrease the demand for energy.

Alghalith, Guo, Wong, and Zhu (2016) present two dynamic models of background risk. We first present a stochastic factor model with an additive background risk. Then, we present a dynamic model of simultaneous (correlated) multiplicative background risk and additive background risk. In so doing, we use a general utility function.

With multiple additive risks, the mean-variance approach and the expected-utility approach of risk preferences are compatible if all attainable distributions belong to the same location-scale 
family. Under this proviso, Guo, Wagener, Wong, and Zhu (2017) survey existing results on the parallels of the two approaches with respect to risk attitudes, the changes thereof, and the comparative statics for simple, linear choice problems under risks.

In the mean-variance approach, all effects can be couched in terms of the marginal rate of substitution between mean and variance. We provide some simple proofs of previous results. We apply the theory we stated or developed in our paper to study the behavior of a banking firm, and examine risk taking behavior with background risk in the mean-variance model.

\section{Statistical and Econometric Models}

Another suggestion is to develop statistical and econometric models in the areas related to management information, decision sciences, economics, and finance. After developing mathematical models, one might consider developing some related statistical and econometric models. We have developed several econometrics papers related to management information, decision sciences, economics, and finance.

\subsection{Testing Investors' behavioral models}

Lam, Liu, and Wong (2008, 2010) developed a Bayesian model to excess volatility, short-term underreaction and long-term overreaction. Guo, McAleer, Wong, and Zhu (2017) extend their model to excess volatility, short-term underreaction and long-term overreaction during financial crises. Fabozzi, Fung, Lam, and Wong (2013) develop three tests to test for the magnitude effect of short-term underreaction and long-term overreaction.

\subsection{Portfolio Optimization}

We have been developing some new theoretical results on portfolio optimization. When the dimension of the data is large, the theoretical model of the classical MV portfolio optimization developed by Markowitz (1952) has been found to have big problem in estimation. As substituting the sample mean and covariance matrix into the MV optimization procedure will 
result in a serious departure of the optimal return estimate, and the corresponding portfolio allocation estimate from their theoretic counterparts when the number of the assets is large, we call this return estimate the "plug-in" return and its corresponding estimate for the asset allocation the "plug-in allocation."

Bai, Liu, and Wong (2009a) prove that this phenomenon is normal and we call it "overprediction." In order to circumvent this over-prediction problem, we use a new method by incorporating the idea of the bootstrap into the theory of large dimensional random matrix. We develop new bootstrap-corrected estimates for the optimal return and its asset allocation, and prove that these bootstrap-corrected estimates can analytically correct the over-prediction and drastically reduce the error. We also show that the bootstrap-corrected estimate of return and its corresponding allocation estimate are proportionally consistent with their counterpart parameters.

Bai, Liu, and Wong (2009a) propose a bootstrap-corrected estimator to correct the overestimation, but there is no closed form for their estimator. Thus, it has to be obtained by employing a bootstrap approach. As a result, it is difficult for practitioners to adopt the estimate in reality. In order to circumvent this limitation, Leung, $\mathrm{Ng}$, and Wong (2012) develop a new estimator for the optimal portfolio return based on an unbiased estimator of the inverse of the covariance matrix and its related terms, and derive explicit formulae for the estimator of the optimal portfolio return.

Li, Bai, McAleer, and Wong (2017) improve estimation by using the spectral distribution of the sample covariance. We develop the limiting behavior of the quadratic form with the sample spectral corrected covariance matrix, and explain the superior performance to the sample covariance as the dimension increases to infinity with the sample size proportionally. Moreover, this paper deduces the limiting behavior of the expected return and risk on the spectral corrected MV portfolio, and illustrates the superior properties of the spectral corrected MV portfolio.

In simulations, we compare the spectrally corrected estimates with the traditional and bootstrapcorrected estimates, and show the performance of the spectral corrected estimates is the best in the portfolio return and also in the portfolio risk. We also compare the performance of our 
proposed estimation with different optimal portfolio estimates for real data from the S\&P 500 . The findings are consistent with the theory developed in our paper.

\subsection{Stochastic Dominance}

Ng, Wong, and Xiao (2017) develop tests for stochastic dominance by translating the inference problem of stochastic dominance into parameter restrictions in quantile regressions are proposed. They are variants of the one-sided Kolmogorov-Smirnoff statistic with a limiting distribution of the standard Brownian bridge. The procedure to obtain the critical values of our proposed test statistics are provided. Simulation results show their superior size and power. They are applied to the NASDAQ 100 and S\&P 500 indices to investigate the dominance relationship before and after major turning points. The results show no arbitrage opportunity between the bear and bull markets. The results infer that markets are inefficient, and risk averters are better off investing in the bull rather than in the bear market.

Bai, Li, McAleer, and Wong (2015) derive the limiting process of stochastic dominance statistics for risk averters as well as for risk seekers when the underlying processes are dependent or independent. We take account of the dependency of the partitions and propose a bootstrap method to decide the critical point. In addition, we illustrate the applicability of the stochastic dominance statistics for both risk averters and risk seekers to analyze the dominance relationship between the Chinese and US stock markets in the entire period, as well as the sub-periods before and after the crises, including the internet bubble and the recent sub-prime crisis.

The findings could be used to draw inferences on the preferences of risk averters and risk seekers in investing in the Chinese and US stock markets. The results also enable us to examine whether there are arbitrage opportunities in these markets, and whether these markets are efficient and investors are rational.

Bai, Li, Liu and Wong (2011) develop new statistics for both PSD and MSD of the first three orders. These statistics provide a tool to examine the preferences of investors with S-shaped utility functions in the prospect theory and investors with RS-shaped investors. We also derive 
the limiting distributions of the test statistics to be stochastic processes. In addition, we propose a bootstrap method to decide the critical points of the tests and prove the consistency of the bootstrap tests. To illustrate the applicability of our proposed statistics, we apply them to study the preferences of investors with the corresponding S-shaped and RS-shaped utility functions vis-a-vis returns on iShares, and vis-a-vis returns of traditional stocks and Internet stocks, before and after the Internet bubble.

\subsection{Risk Measures}

Leung and Wong (2008) apply the technique of the repeated measures design to develop the Multiple Sharpe ratio test statistic to test the hypothesis of the equality of the multiple Sharpe ratios. We also solve the asymptotic distribution of the statistic and its properties. To demonstrate the superiority of our proposed statistic over the traditional pair-wise Sharpe ratio test, we illustrate our approach by testing the equality of Sharpe ratios for eighteen iShares. Whereas the pair-wise Sharpe ratio test show that the performance of all the 18 iShares are indistinguishable, our test results reject the equality of the Sharpe ratios in each year, as well as in the entire sample, implying that the 18 iShares perform differently in each year as well as in the entire sample, with some outperforming others in the market.

Bai, Wang, and Wong (2011) develop the mean-variance-ratio statistic to test the equality of the mean-variance ratios and prove that our proposed statistic is uniformly most powerful unbiased. In addition, we illustrate the applicability of our proposed test to compare the performances of stock indices.

Bai, Hui, Wong, and Zitikis (2012) propose and develop mean-variance-ratio (MVR) statistics for comparing the performance of prospects after the effect of the background risk has been mitigated. We investigate the performance of the statistics in large and small samples and show that in the non-asymptotic framework, the MVR statistic produces a uniformly most powerful unbiased (UMPU) test. We discuss the applicability of the MVR test in the case of large samples and illustrate its superiority in the case of small samples by analyzing Korea and Singapore stock returns after the impact of the American stock returns (which we view as the background risk) 
has been deducted. We find, in particular, that when samples are small, the MVR statistic can detect differences in asset performances, while the Sharpe ratio test, which is the mean-standarddeviation-ratio statistic, may not be able to do so.

\subsection{Unit root, cointegration, and causality tests}

We have developed several tests related to unit roots, cointegration, and causality, and discuss a few here.

Tiku and Wong (1998) develop a unit root test to accommodate data that follow an AR(1) model. We use the three-moment chi-square and four moment $\mathrm{F}$ approximations to testing a unit root in the AR(1) model when the innovations have one of a very wide family of symmetric distribution Student t.

In cointegration theory, vector error-correction models (VECMs) have become an important means of analysing cointegrating relations. Usual full-order VECMs assume all nonzero entries in their coefficient matrices. However, applications of VECMs to economic and financial time series data have revealed that zero entries are indeed possible. If indirect causality or Granger non-causality exists among the variables, the use of a full-order VECM will incorrectly conclude only the existence of Granger causality among these variables.

In addition, the statistical and numerical accuracy of the cointegrating vectors estimated in this misspecified full-order VECM will be doubtful. It is argued that the zero-non-zero (ZNZ) patterned VECM is a more straightforward and an effective means of testing for both indirect causality and Granger non-causality. Penm, Terrell, and Wong (2003) present simulations and an application that demonstrates the usefulness of the ZNZ patterned VECM.

Lam, Wong, and Wong (2006) develop some properties on the autocorrelation of the k-period returns for the general mean reversion (GMR) process in which the stationary component is not restricted to the AR(1) process, but takes the form of a general ARMA process. We then derive some properties of the GMR process and three new nonparametric tests that compare the relative 
variability of returns over different horizons to validate the GMR process as an alternative to random walk. We examine the asymptotic properties of these tests, which can then be applied to identify random walk models from the GMR processes.

The traditional linear Granger causality test has been widely used to examine linear causality among several time series in bivariate settings, as well as multivariate settings. Hiemstra and Jones (1994) develop a nonlinear Granger causality test in a bivariate setting to investigate the nonlinear causality between stock prices and trading volume. Bai, Wong, and Zhang (2010) extend their work by developing a nonlinear causality test in multivariate settings. Bai, Li, Wong, and Zhang (2011) discuss linear causality tests in multivariate settings, and thereafter develop a nonlinear causality test in multivariate settings. A Monte Carlo simulation is conducted to demonstrate the superiority of our proposed multivariate test over its bivariate counterpart. In addition, we illustrate the applicability of our proposed test to analyze the relationships among different Chinese stock market indices.

Hui, Wong, Bai, and Zhu (2017) propose a quick and efficient method to examine whether a time series $\mathrm{Y}$ possesses any nonlinear feature by testing a kind of dependence remained in the residuals after fitting $\mathrm{Y}$ with a linear model. The advantage of the proposed nonlinearity test is that it is not required to know the exact nonlinear features and the detailed nonlinear forms of $\mathrm{Y}$. It can also be used to test whether the hypothesized model, including linear and nonlinear, to the variable being examined is appropriate as long as the residuals of the model being used can be

estimated. A simulation study shows that the proposed test is stable and powerful. We apply the proposed statistic to test whether there is any nonlinear feature in the sunspot data and whether the S\&P 500 index follows a random walk model. The conclusion drawn from the proposed test is consistent with those from other tests.

\subsection{Other Econometric Models/Tests}

We have developed some other econometric/statistic models/tests, and list a few. 
Wong and Miller (1990) develop a theory and methodology for repeated time series (RTS) measurements on autoregressive integrated moving average-noise (ARIMAN) process. The theory enables us to relax the normality assumption in the ARIMAN model and to identify models for each component series of the process. We discuss the properties, estimation, and forecasting of RTS ARIMAN models and illustrate with examples. Wong, Miller, and Shrestha (2001) extend their work by allowing the error variance as well as the number of repetition to change over time. They show that the model is identified, and derive the maximum likelihood estimator using the Kalman filter technique.

Tiku, Wong, Vaughan, and Bian (2000) consider AR $(q)$ models in time series with non-normal innovations represented by a member of a wide family of symmetric distributions (Student $t$ ). As the ML (maximum likelihood) estimators are intractable, we derive the MML (modified maximum likelihood) estimators of the parameters and show that they are remarkably efficient. We use these estimators for hypothesis testing, and show that the resulting tests are robust and powerful.

Tiku, Wong, and Bian (1999a) extend the work by considering AR(q) models in time series with asymmetric innovations represented by two families ofdistributions: (i) gamma with support IR: $(0, \infty)$; and (ii) generalized logistic with support IR: $(-\infty, \infty)$. As the ML (maximum likelihood) estimators are intractable, we derive the MML (modified maximum likelihood) estimators of the parameters and show that they are remarkably efficient, besides being easy to compute. We investigate the efficiency properties of the classical LS (least squares) estimators. Their efficiency relative to the proposed MML estimators is very low.

Tiku, Wong, and Bian (1999b) estimate coefficients in a simple regression model with autocorrelated errors. The underlying distribution is assumed to be symmetric, one of the Student t family for illustration. Closed-form estimators are obtained and shown to be remarkably efficient and robust. Wong and Bian (2005) extend the results to where the underlying distribution is a generalized logistic distribution. The generalized logistic distribution family represents very wide skewed distributions, ranging from highly right skewed to highly left skewed. Analogously, we develop the MML estimators as the ML (maximum likelihood) 
estimators are intractable for the generalized logistic data. We then study the asymptotic properties of the proposed estimators and conduct simulations.

Bian and Dickey (1996) develop a robust Bayesian estimator for the vector of regression coefficients using a Cauchy-type g-prior. This estimator is an adaptive weighted average of the least squares estimator and the prior location, and is of great robustness with respect to fat-tailed sample distribution. Wong and Bian (2000) introduce the robust Bayesian estimator to the estimation of the Capital Asset Pricing Model (CAPM) in which the distribution of the error component is well-known to be fat-tailed.

To support the proposal, we apply both the robust Bayesian estimator and the least squares estimator in the simulation of the CAPM and in the analysis of the CAPM for US annual and monthly stock returns. The simulation results show that the Bayesian estimator is robust and superior to the least squares estimator when the CAPM is contaminated by large normal and/or non-normal disturbances, especially by Cauchy disturbances.

In the empirical study, we find that the robust Bayesian estimate is uniformly more efficient than the least squares estimate in terms of the relative efficiency of one-step ahead forecast mean square error, especially for small samples. Bian, McAleer, and Wong (2013) develop a modified maximum likelihood (MML) estimator for the multiple linear regression model with underlying student $\mathrm{t}$ distribution.

We obtain the closed form of the estimators, derive the asymptotic properties, and demonstrate that the MML estimator is more appropriate for estimating the parameters of the Capital Asset Pricing Model (CAPM) by comparing its performance with least squares estimators (LSE) on the monthly returns of US portfolios. The empirical results reveal that the MML estimators are more efficient than LSE in terms of the relative efficiency of one-step-ahead forecast mean square error in small samples.

Bian, McAleer, and Wong (2011) develop a new test, the trinomial test, for pairwise ordinal data samples to improve the power of the sign test by modifying its treatment of zero differences 
between observations, thereby increasing the use of sample information. Simulations demonstrate the power superiority of the proposed trinomial test statistic over the sign test in small samples in the presence of tie observations. We also show that the proposed trinomial test has substantially higher power than the sign test in large samples and also in the presence of tie observations, as the sign test ignores information from observations resulting in ties.

Homm and Pigorsch (2012) use the Aumann and Serrano index to develop a new economic performance measure (EPM), which is well known to have advantages over other measures. Niu, Guo, McAleer, and Wong (2017) extend the theory by constructing a one-sample confidence interval of EPM, and construct confidence intervals for the difference of EPMs for two independent samples. We also derive the asymptotic distribution for EPM and for the difference of two EPMs when the samples are independent, and conduct simulations to show the proposed theory performs well for one and two independent samples. The simulations show that the proposed approach is robust in the dependent case. The theory developed is used to construct both one-sample and two-sample confidence intervals of EPMs for Singapore and USA stock indices.

\section{Empirical Studies}

Another suggestion is to apply statistical and econometric models to examine the relationships among the variables in some important issues in management information, decision sciences, and financial economics. We obtain several important applications of the theories developed. In order to save space, we skip our discussion here. Readers may refer to Chang, McAleer, and Wong (2016a, b, c) for further information.

\section{Concluding Remarks}

In this paper, we discussed different types of utility functions, stochastic dominance, mean-risk models, portfolio optimization, and cointegration and causality, as these topics are popular in management information, decision sciences, and financial economics in terms of theory and econometric analysis. Authors could also extend their work to link the three disciplines. 
Although we have discussed the contributions in $\mathrm{SD}$, MR, and PO related to management information, decision sciences, and financial economics, there are theoretical contributions in other areas that could also be useful in these disciplines. Readers may refer to Chang, McAleer, and Wong $(2016 \mathrm{a}, \mathrm{b})$ for contributions in related cognate areas that might be useful in management science, economics, and finance. 


\section{References}

Alghalith, M., Guo, X., Wong, W.K., Zhu, L.X., 2016, A General Optimal Investment Model in the Presence of Background Risk, Annals of Financial Economics 11(1), 1650001.

Alghalith, M., Guo, X., Niu, C.Z., Wong, W.K., 2017, Input Demand under Joint Energy and Output Prices Uncertainties, Asia Pacific Journal of Operational Research, 34, 1750018.

Alghalith, M., Niu, C.Z., Wong, W.K., 2017, The Impacts of Joint Energy and Output Price Uncertainties in a Mean-Variance Framework, Theoretical Economics Letters 7(5), 1108-1120.

Bai, Z.D., Hui, Y.C., Wong, W.K., Zitikis, R., 2012. Prospect Performance Evaluation: Making a Case for a Non-asymptotic UMPU Test, Journal of Financial Econometrics, 10(4), 703-732.

Bai, Z.D., Guo, X., Li, H., Wong, W.K., 2017, Stochastic Dominance with Applications in Economics, Finance, and Income Inequality, forthcoming.

Bai, Z.D., Hui, Y.C., Wong, W.K., Zitikis, R., 2012, Evaluating Prospect Performance: Making a Case for a Non-Asymptotic UMPU Test, Journal of Financial Econometrics, 10(4), 703-732.

Bai, Z.D., Li, H., Liu, H.X., Wong, W.K., 2011, Test Statistics for Prospect and Markowitz Stochastic Dominances with Applications, Econometrics Journal, 14(2), 278-303.

Bai, Z.D., Li, H., McAleer, M., Wong, W.K., 2015, Stochastic Dominance Statistics for Risk Averters and Risk Seekers: An Analysis of Stock Preferences for USA and China, Quantitative Finance, 15(5), 889-900.

Bai, Z.D., Li, H., Wong, W.K., Zhang, B.Z., 2011, Multivariate Causality Tests with Simulation and Application, Statistics and Probability Letters, 81(8), 1063-1071.

Bai, Z.D., Liu, H.X., Wong, W.K., 2009a, Enhancement of the Applicability of Markowitz's Portfolio Optimization by Utilizing Random Matrix Theory, Mathematical Finance 19(4), 639667.

Bai, Z.D., Lui, H.X., Wong, W.K., 2009b, On the Markowitz Mean-Variance Analysis of SelfFinancing Portfolios, Risk and Decision Analysis, 1(1), 35-42.

Bai, Z.D., Wong, W.K., Zhang, B.Z., 2010, Multivariate Linear and Nonlinear Causality Tests, Mathematics and Computers in Simulation, 81, 5-17.

Bai, Z.D., Li, H., Liu, H.X., Wong, W.K., 2011, Test Statistics for Prospect and Markowitz Stochastic Dominance with Applications, Econometrics Journal, 122, 1-26. 
Bai, Z.D., Li, H., McAleer, M., Wong, W.K., 2015, Stochastic Dominance Statistics for Risk Averters and Risk Seekers: An Analysis of Stock Preferences for USA and China, Quantitative Finance, 15(5), 889-900.

Bai, Z.D., Li, H., McAleer, M., Wong, W.K., 2016, Spectrally-corrected Estimation for Highdimensional Markowitz Mean-Variance Optimization, Tinbergen Institute Discussion Paper, TI 2016-025/III.

Bai, Z.D., Liu, H.X., Wong, W.K., 2009a, Enhancement of the Applicability of Markowitz's Portfolio Optimization by Utilizing Random Matrix Theory, Mathematical Finance, 19(4), 639667.

Bai, Z.D., Liu, H.X., Wong, W.K., 2009b, On the Markowitz Mean-Variance Analysis of Selffinancing Portfolios, Risk and Decision Analysis, 1(1), 35-42.

Bai, Z.D., Wang, K.Y., Wong, W.K., 2011, Mean-variance Ratio Test, a Complement to Coefficient of Variation Test and Sharpe Ratio Test, Statistics and Probability Letters, 81(8), 1078-1085.

Bawa, V.S., Bodurtha Jr, J.N., Rao, M.R., Suri, H.L., 1985, On Determination of Stochastic Dominance Optimal Sets, Journal of Finance, XL, 2, 417-431.

Bian, G., Dickey, J.M., 1996, Properties of Multivariate Cauchy and Poly-Cauchy Distributions with Bayesian g-prior Applications. in Bayesian Analysis in Statistics and Econometrics: Essay in Honor of Arnold Zellner, edited by Berry, D.A., Chaloner, K.M., Geweke, J.K., Wiley, New York, 299-310.

Bian, G., McAleer, M., Wong, W.K., 2013, Robust Estimation and Forecasting of the Capital Asset Pricing Model, Annals of Financial Economics, 1350007, DOI: 10.1142/S2010495213500073.

Broll, U., Egozcue, M., Wong, W.K., Zitikis, R., 2010, Prospect Theory, Indifference Curves, and Hedging Risks, Applied Mathematics Research Express, 2010(2), 142-153.

Broll, U., Guo, X., Welzel, P., Wong, W.K., 2015, The Banking Firm and Risk Taking in a TwoMoment Decision Model, Economic Modelling, 50, 275-280.

Broll, U., Wahl, J.E., Wong, W.K., 2006, Elasticity of Risk Aversion and International Trade, Economics Letters, 91(1), 126-130.

Broll, U., Wong, W.K., Wu, M., 2011, Banking Firm, Risk of Investment and Derivatives. Technology and Investment, 2, 222-227.

Chan, R.H., Clark, E., Wong, W.K., 2012, On the Third Order Stochastic Dominance for RiskAverse and Risk-Seeking Investors, MPRA Paper 42676, University Library of Munich, Germany. 
Chang, C.-L., McAleer, M., Wong, W.K., 2016a, Behavioural, Financial, and Health \& Medical Economics: A connection, Journal of Health \& Medical Economics, 2(1:1), 1-4.

Chang, C.-L., McAleer, M., Wong, W.K., 2016b, Informatics, Data Mining, Econometrics and Financial Economics: A connection, Journal of Informatics and Data Mining, 1(1:7), 1-5.

Chang, C.-L., McAleer, M., Wong, W.K., 2016c, Management Science, Economics and Finance: A Connection, International Journal of Economics and Management Sciences, 5(4), 1-19.

Egozcue, M., Wong, W.K., 2010, Gains from Diversification: A Majorization and Stochastic Dominance Approach, European Journal of Operational Research, 200(3), 893-900.

Egozcue, M., Fuentes García, L., Wong, W.K., Zitikis, R., 2011, Do Investors like to Diversify? A Study of Markowitz Preferences, European Journal of Operational Research, 215(1), 188-193.

Fabozzi, F.J., Fung, C.Y., Lam, K., Wong, W.K., 2013, Market Overreaction and Underreaction: Tests of the Directional and Magnitude Effects, Applied Financial Economics, 23(18), 14691482 .

Farinelli, S., Tibiletti, L., 2008, Sharpe Thinking in Asset Ranking with One-sided Measures, European Journal of Operational Research, 185 (3), 1542-1547.

Fishburn, P.C., 1974, Convex stochastic dominance with continuous distribution functions. Journal of Economic Theory, 7, 143-158.

Fung, E.S., Kin Lam, K., Siu, T.K., Wong, W.K., 2011, A New Pseudo Bayesian Model for Financial Crisis, Journal of Risk and Financial Management, 4, 42-72.

Gordon, M.J., E. Shapiro, 1956, Capital Equipment Analysis: The Required Rate of Profit,,Management Science, X, 102-110.

Guo, X., Jiang, X.J., Wong, W.K., 2017, Stochastic Dominance and Omega Ratio: Measures to Examine Market Efficiency, Arbitrage Opportunity, and Anomaly, Economies, forthcoming,

Guo, X., Levy, H., Wong, W.K., 2015, Consistent Tests for Almost Stochastic Dominance, Social Science Research Network Working Paper Series 2592205.

Guo, X., Lien, D., Wong, W.K., 2016, Good Approximation of Exponential Utility Function for Optimal Futures Hedging, Journal of Mathematical Finance 6, 457-463.

Guo, X., McAleer, M., Wong, W.K., Zhu, L.X., 2017, A Bayesian Approach to Excess Volatility, Short-term Underreaction and Long-term Overreaction during Financial Crises, North American Journal of Economics and Finance, 42, 346-358. 
Guo, X., Post, T., Wong, W.K., Zhu, L.X., 2014, Moment Conditions for Almost Stochastic Dominance, Economics Letters, 124(2), 163-167.

Guo, X., Qiao, Z., Wong, W.K., 2017, Why Investors Buy Insurance and Try their Luck with Lotteries as well?, Social Science Research Network, Working Paper Series 2980007.

Guo, X., Wagener, A., Wong, W.K., Zhu, L.X., 2017), The Two-Moment Decision Model with Additive Risks, Risk Management, forthcoming.

Guo, X., Wong, W.K., 2016, Multivariate Stochastic Dominance for Risk Averters and Risk Seekers, RAIRO - Operations Research, 50(3), 575-586.

Guo, X., Wong, W.K., Zhu, L.X., 2016, Almost Stochastic Dominance for Risk Averters and Risk Seekers, forthcoming in Finance Research Letters.

Guo, X., Zhu, X.H., Wong, W.K., Zhu, L.X., 2013, A Note on Almost Stochastic Dominance, Economics Letters, 121(2), 252-256.

Homm, U. and Pigorsch, C., 2012, Beyond the Sharpe Ratio: An Application of the AumannSerrano Index to Performance Measurement, Journal of Banking \& Finance, 36, 2274-2284.

Leshno, M., Levy, H., 2002, Preferred by "All" and Preferred by "Most" Decision Makers: Almost Stochastic Dominance, Management Science, 48(8), 1074-1085.

Lam, K., Liu, T.S., Wong, W.K., 2010, A Pseudo-Bayesian Model in Financial Decision Making with Implications to Market Volatility, Under- and Over-reaction, European Journal of Operational Research, 203(1),166-175.

Lam, K., Liu, T.S., Wong, W.K., 2012, A New Pseudo Bayesian Model with Implications to Financial Anomalies and Investors' Behavior, Journal of Behavioral Finance, 13(2), 93-107.

Leung, P.L., Wong, W.K., 2008, On Testing the Equality of the Multiple Sharpe Ratios, with Application on the Evaluation of Ishares, Journal of Risk, 10(3), 1-16.

Levy, H., 2015, Stochastic Dominance: Investment Decision Making under Uncertainty, $3^{\text {rd }}$ Edition, Springer, New York.

Levy, H., M. Levy, 2004, Prospect Theory and Mean-variance Analysis, Review of Financial Studies, 17(4), 1015-1041.

Levy, M., H. Levy, 2002, Prospect Theory: Much Ado about Nothing?, Management Science, 48(10), 1334-1349.

Leung, P.L., Ng, H.Y., Wong, W.K., 2012, An Improved Estimation to Make Markowitz's Portfolio Optimization Theory User Friendly and Estimation Accurate with Application on the US Stock Market Investment, European Journal of Operational Research, 222(1), 85-95. 
Li, H., Z.D. Bai, M. McAleer, W.K. Wong, 2017, Spectrally-Corrected Estimation for HighDimensional Markowitz Mean-Variance Optimization, working paper.

Li, C.K., W.K. Wong, 1999, Extension of Stochastic Dominance Theory to Random Variables, RAIRO - Operations Research, 33(4), 509-524.

Lien, D., 2008, Optimal Futures Hedging: Quadratic versus Exponential Utility Functions, Journal of Futures Markets, 28, 208-211.

Lozza, S.O., Wong, W.K., Fabozzi, F.J., Egozcue, M., 2017, Behavioral Explanation to the Diversification Puzzle, working paper.

Ma, C., Wong, W.K., 2010, Stochastic Dominance and Risk Measure: A Decision-theoretic Foundation for VaR and C-VaR, European Journal of Operational Research, 207(2), 927-935.

Markowitz, H.M., 1952a, The Utility of Wealth, Journal of Political Economy, 60, 151-156.

Markowitz, H.M., 1952b, Portfolio Selection, Journal of Finance, 7, 77-91.

Ng, P., Wong, W.K., Xiao, Z.J., 2017, Stochastic Dominance Via Quantile Regression, European Journal of Operational Research, 261(2), 666-678.

Niu, C.Z., Guo, X., McAleer, M., Wong, W.K., 2017, Theory and Application of an Economic Performance Measure of Risk, International Review of Economics \& Finance, first revision.

Niu, C.Z., Wong, W.K., Xu, Q.F., 2017, Kappa Ratios and (Higher-Order) Stochastic Dominance, Risk Management, 19(3), 245-253.

Sriboonchitta, S., W.K. Wong, S. Dhompongsa, H.T. Nguyen, 2009, Stochastic Dominance and Applications to Finance, Risk and Economics, Chapman and Hall/CRC, Taylor and Francis, Boca Raton, Florida, USA.

Thompson, H.E., 1985, The Magnitude and Reliability of Equity Capital Cost Estimates: A Statistical Approach, Managerial and Decision Economics, 6, 132-140.

Thompson, H.E., W.K. Wong, 1991, On the Unavoidability of "Unscientific"' Judgement in Estimating the Cost of Capital, Managerial and Decision Economics, 12, 27-42.

Thompson, H.E., W.K. Wong, 1996, Revisiting 'Dividend Yield Plus Growth' and Its Applicability, Engineering Economist, 41(2), 123-147.

Tiku, M.L., Wong, W.K., 1998, Testing for Unit Root in AR(1) Model Using Three and Four Moment Approximations, Communications in Statistics: Simulation and Computation, 27(1), 185-198. 
Tiku, M.L., Wong W.K., Bian, G., 1999a, Time Series Models with Asymmetric Innovations, Communications in Statistics: Theory and Methods, 28(6), 1331-1360.

Tiku, M.L., Wong W.K., Bian, G., 1999b, Estimating Parameters in Autoregressive Models in Non-normal Situations: Symmetric Innovations, Communications in Statistics: Theory and Methods, 28(2), 315-341.

Tiku, M.L., Wong, W.K., Vaughan, D.C., Bian, G., 2000, Time series models in non-normal situations: Symmetric innovations, Journal of Time Series Analysis, 21, 571-96.

Tsetlin, I., Winkler, R.L., Huang, R.J., Tzeng, L.Y., 2015. Generalized Almost Stochastic Dominance. Operations Research. 62(2), 363-377

Tzeng, L.Y., Huang, R.J., Shih, P.-T., 2013, Revisiting Almost Second-degree Stochastic Dominance, Management Science, 59, 1250-1254.

Wong, W.K., 2007, Stochastic Dominance and Mean-variance Measures of Profit and Loss for Business Planning and Investment, European Journal of Operational Research, 182(2), 829-843.

Wong, W.K., Chan, R., 2008, Markowitz and Prospect Stochastic Dominances, Annals of Finance, 4(1), 105-129.

Wong, W.K., Bian, G., 2000, Robust Bayesian Inference in Asset Pricing Estimation, Journal of Applied Mathematics and Decision Sciences, 4(1), 65-82.

Wong, W.K., Bian, G., 2005, Estimating Parameters in Autoregressive Models with Asymmetric Innovations, Statistics and Probability Letters, 71(1), 61-70.

Wong, W.K., C.K. Li., 1999, A Note on Convex Stochastic Dominance Theory, Economics Letters, 62, 293-300.

Wong, W.K., Miller, R.B., 1990, Repeated Time Series Analysis of ARIMA-Noise Models", Journal of Business and Economic Statistics, 8(2), 243-250.

Wong, W.K., Miller, R.B., Shrestha, K., 2001, Maximum Likelihood Estimation of ARMA Model with Error Processes for Replicated Observation, Journal of Applied Statistical Science, 10(4), 287-297.

Wong, W.K., Ma, C., 2008, Preferences over Location-scale Family, Economic Theory, 37(1), 119-146.

W.K. Wong, J.A. Wright, S.C.P. Yam, S.P. Yung, 2012, A Mixed Sharpe Ratio, Risk and Decision Analysis, 3(1-2), 37-65. 
